\title{
Ruptura pós-emética do esôfago: a síndrome de Boerhaave*
}

\author{
Postemetic rupture of the esophagus: Boerhaave's syndrome
}

Henrique José da Mota ${ }^{1}$, Manoel Ximenes Netto $^{2}$, Aldo da Cunha Medeiros ${ }^{3}$

\section{Resumo}

A ruptura pós-emética do esôfago, também chamada ruptura espontânea ou síndrome de Boerhaave, foi descrita pela primeira vez em 1724 por Herman Boerhaave. Trata-se de uma doença grave, de alta mortalidade e de difícil diagnóstico, tanto por ser rara como por ser freqüentemente confundida com quadros graves mais comuns, como o infarto agudo do miocárdio, a úlcera péptica perfurada e a pancreatite aguda. Descrevemos, a seguir, três casos de pacientes com esta síndrome. Dois foram submetidos ao reparo primário da lesão e um foi submetido à esofagectomia com posterior reconstrução. Houve um óbito por choque séptico no pós-operatório imediato. Os outros dois casos tiveram boa evolução a longo prazo.

Descritores: Esofagopatias; Ruptura espontânea; Mediastinite; Esofagectomia.

\begin{abstract}
Postemetic rupture of the esophagus, also known as spontaneous rupture or Boerhaave's syndrome, was first described by Herman Boerhaave in 1724. This is a severe disease that causes high mortality rates and is difficult to diagnose not only because it is rare but also because it is frequently confused with other severe clinical conditions, such as acute myocardial infarction, perforated peptic ulcer, and acute pancreatitis. Herein, we describe three cases of patients with this syndrome. Two underwent primary repair of the rupture, and one underwent esophagectomy followed by reconstruction. There was one death due to septic shock in the immediate postoperative period. The other two patients presented favorable long-term evolution.
\end{abstract}

Keywords: Esophageal diseases; Rupture, spontaneous; Mediastinitis; Esophagectomy.

\section{Introdução}

A ruptura pós-emética do esôfago torácico, conhecida como síndrome de Boerhaave, foi descrita pela primeira vez por Herman Boerhaave em 1724 no relato do caso do almirante da esquadra holandesa Barão Jan von Wassenaer, cuja morte foi ocasionada por repetidos episódios de vômito auto-induzido. 0 primeiro reparo bem sucedido desta condição ocorreu somente em 1947 e foi relatado por Norman Barrett em Londres. ${ }^{(1)}$

Trata-se de um quadro clínico extremamente grave em decorrência da mediastinite e da sepse, que rapidamente se instala, ocasionada pelo extravasamento de secreções digestivas e alimentos para o mediastino e espaço pleural. 0 seu diagnóstico é freqüentemente retardado pelo fato de a doença ser rara e ser geralmente confundida com outras patologias igualmente graves porém mais prevalentes, como o infarto agudo do miocárdio, a úlcera péptica perfurada e a pancreatite aguda, dentre outras.(1) Tudo isto contribui para a sua elevada mortalidade, em torno de 39\%, apesar dos recursos médicos atuais. ${ }^{(2)}$ Relatamos, a seguir, três casos tratados recentemente pelos autores.

\section{Relato dos casos}

\section{Caso 1}

Um paciente do sexo masculino com 49 anos de idade foi internado na Unidade de Emergência do Hospital

\footnotetext{
* Trabalho realizado no Departamento de Cirurgia do Centro de Ciências da Saúde da Universidade Federal do Rio Grande do Norte - CCS/UFRN - Natal (RN) Brasil; Hospital Walfredo Gurgel da Secretaria de Saúde Pública do Estado do Rio Grande do Norte, Natal (RN) Brasil; Clínica do Tórax do Hospital Santa Lúcia, Brasília (DF) Brasil.

1. Título de especialista pela Sociedade Brasileira de Cirurgia Torácica, Universidade Federal do Rio Grande do Norte - UFRN - Natal (RN) Brasil.

2. Cirurgião Torácico do Hospital Santa Lúcia, Brasilia (DF) Brasil.

3. Professor Titular do Departamento de Cirurgia do Centro de Ciências da Saúde da Universidade Federal do Rio Grande do Norte - CCS/UFRN - Natal (RN) Brasil. Endereço para correspondência: Henrique José da Mota. Pneumocentro, Av. Campo Sales, 762, Tirol, CEP 59020-300, Natal, RN, Brasil.

Tel 5584 3222-0303. Fax 5584 3221-2280. E-mail: hjmota@ufrnet.br

Recebido para publicação em 11/6/06. Aprovado, após revisão, em 9/8/06.
} 
Walfredo Gurgel para correção cirúrgica de hérnia umbilical complicada por obstrução intestinal. Aproximadamente $10 \mathrm{~h}$ após a correção cirúrgica, o paciente evoluiu com precordialgia intensa, sudorese, hipotensão e dispnéia, e seu caso foi tratado inicialmente como infarto agudo do miocárdio. Uma radiografia do tórax (Figura 1) evidenciou derrame pleural à esquerda, o que despertou a hipótese inicial de embolia pulmonar. Uma toracocentese revelou líquido escuro, sugerindo secreção gástrica de estase na cavidade pleural esquerda. No interrogatório dirigido, o paciente revelou ter vomitado excessivamente durante toda a internação. A esofagoscopia revelou extenso comprometimento do lúmen distal do esôfago, de onde fluía uma grande quantidade de secreção semelhante à obtida por meio da toracocentese, impedindo a visualização apropriada da lesão.

Com o diagnóstico inicial de ruptura esofágica, o paciente foi submetido à toracotomia póstero-lateral esquerda por meio da qual encontramos extenso comprometimento do esôfago distal com necrose e mediastinite grave. Devido à impossibilidade de reparo primário, optou-se pela esofagectomia subtotal, a esofagostomia cervical, a drenagem pleural, seguidas pela laparotomia e a gastrostomia.

0 pós-operatório foi complicado por insuficiência respiratória, insuficiência renal aguda e

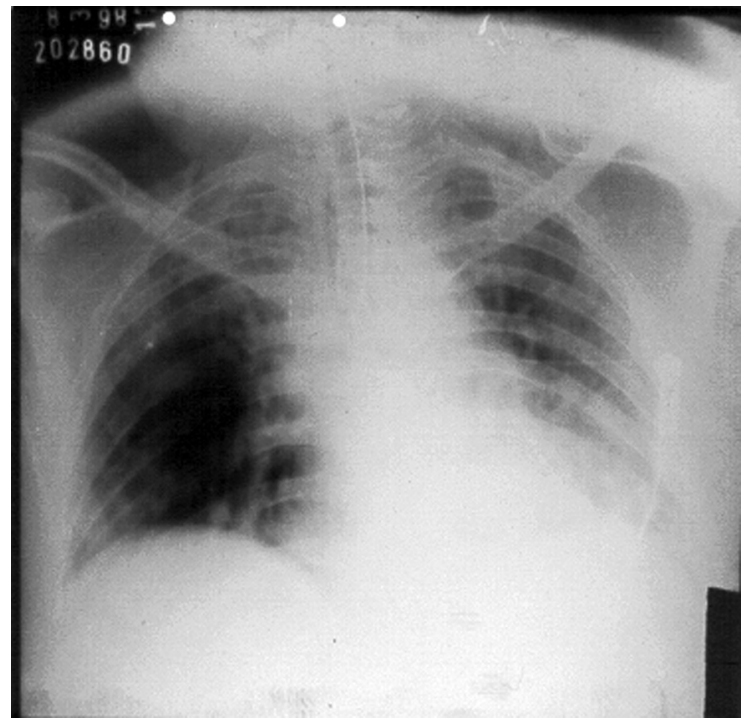

Figura 1 - Radiografia do tórax, com o paciente em decúbito dorsal, mostrando velamento sugestivo de derrame pleural na base do hemitórax esquerdo (Caso 1). empiema pleural que requereu re-operação. 0 paciente necessitou de suporte nutricional parenteral e terapia intensiva durante trinta dias, após os quais recebeu alta hospitalar com descontinuidade faringo-gástrica. Nove meses depois, foi submetido, com sucesso, à reconstrução do trânsito esofágico com tubo gástrico invertido.

\section{Caso 2}

Um paciente do sexo masculino com 38 anos de idade foi admitido na Unidade de Emergência do Hospital Walfredo Gurgel por apresentar enfisema subcutâneo aparentemente espontâneo. No interrogatório dirigido, o paciente relatou alcoolismo e vômitos pós-alimentares recentes. Não havia queixas de dor torácica acentuada, mal-estar ou desconforto respiratório na avaliação inicial. 0 exame físico e a radiografia do tórax revelaram acentuado enfisema subcutâneo no tórax e pescoço e condensação sugestiva de derrame pleural na base esquerda (Figura 2). A toracocentese deu saída a líquido de cor escura e com aspecto sugestivo de conteúdo gástrico, levantando a suspeita de ruptura esofágica, a qual foi confirmada pelo esofagograma.

A toracotomia revelou mediastinite extensa e ruptura linear da parede lateral distal do esôfago. Realizou-se o reparo da lesão usando fio de sutura de

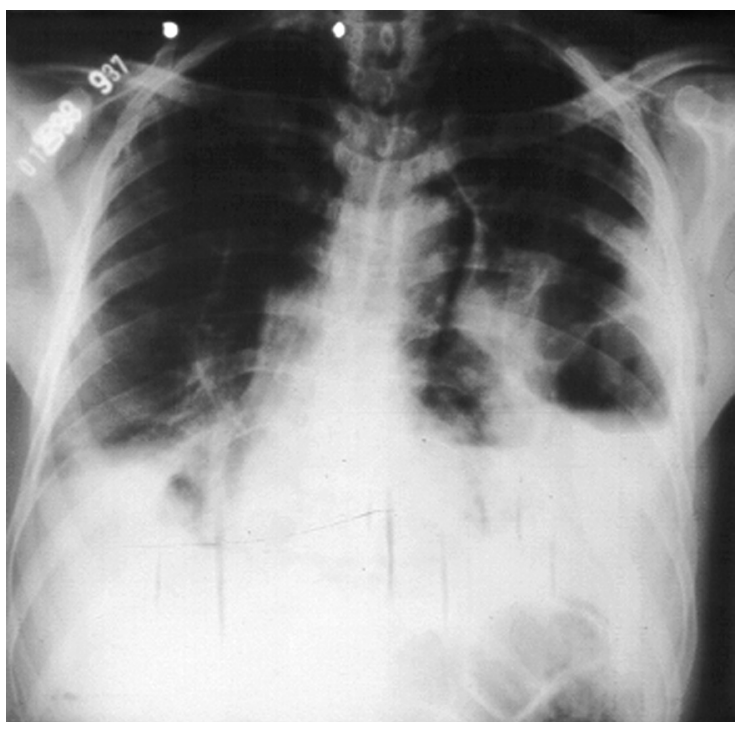

Figura 2 - Radiografia do tórax mostrando pneumomediastino associado a hidropneumotórax à esquerda (Caso 2). 
polipropileno 3-0 (Prolene ${ }^{\circledR}$; Ethicon Endo-surgery, Johnson \& Johnson) com pontos separados, assim como reforço pleural, drenagem pleural ampla e gastrostomia alimentar. No dia seguinte, entretanto, o paciente evoluiu com choque refratário, falência orgânica múltipla e óbito.

\section{Caso 3}

Um paciente do sexo masculino com 72 anos de idade foi admitido na unidade de terapia intensiva de outra instituição com quadro de dor tóracoabdominal após vômitos incessantes e de grande intensidade. A radiografia de tórax inicial mostrou derrame pleural esquerdo. As enzimas cardíacas e o resultado do eletrocardiograma eram normais. A tomografia computadorizada de tórax mostrou derrame pleural moderado e pequeno pneumotórax. 0 paciente apresentava palidez cutânea, dispnéia, mucosas hipocoradas e pulsos periféricos presentes e de boa qualidade. A pressão arterial do paciente era 134/87 mm Hg, a freqüência cardíaca, $75 \mathrm{bpm}$ e a temperatura, $36,8{ }^{\circ} \mathrm{C}$. 0 paciente também apresentava murmúrio vesicular diminuído à esquerda e abdômen doloroso e distendido, sem edemas. A toracocentese mostrou líquido sanguinolento, presença de germes Gram-positivos e amilase elevada de $1.112 \mathrm{U} / \mathrm{dL}$ (normal 60-180 U/dL). 0 tratamento inicial constou em hidratação venosa, analgesia com morfina, antibioticoterapia e passagem de sonda nasogástrica, a qual drenou $1.500 \mathrm{~mL}$ de conteúdo gástrico. 0 paciente foi transferido para o nosso hospital e submetido à esofagoscopia a qual mostrou ruptura esofágica na altura da junção esofagogástrica. Foi realizada toracotomia pelo $6^{\circ}$ espaço intercostal. Encontrou-se grande quantidade de líquido gástrico e ruptura de esôfago distal (Figura 3). Foi efetuada sutura esofágica, seguida de enxerto pediculado de diafragma. A cavidade pleural foi exaustivamente lavada e o dreno pleural sob selo d'água foi posicionado. 0 pós-operatório não foi complicado e, dois anos e 9 meses depois, o paciente encontra-se assintomático e exercendo suas atividades profissionais.

\section{Discussão}

A perfuração do esôfago torácico é uma das mais sérias lesões do trato digestivo em termos de mortalidade e morbidade. Ocasiona um grave quadro de mediastinite e sepse devido ao extravasamento de

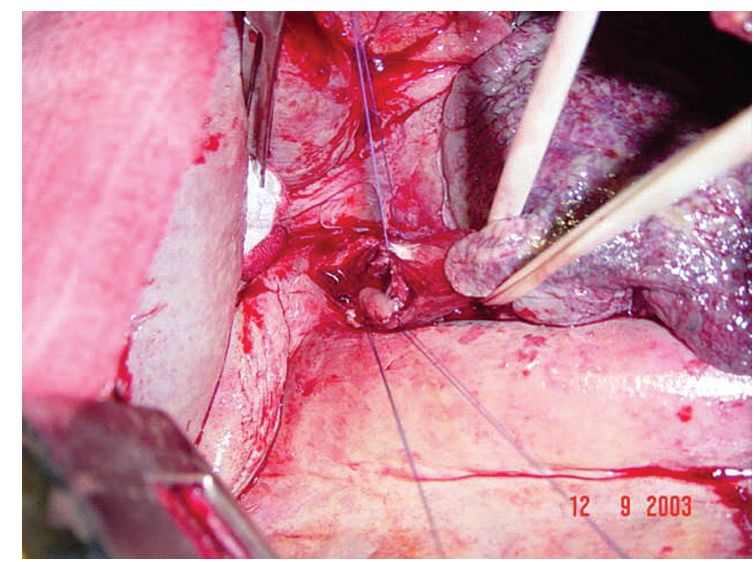

Figura 3 - Fotografia operatória mostrando perfuração do esôfago distal (Caso 3).

secreções digestivas e partículas alimentares para o mediastino e, eventualmente, para o espaço pleural. 0 prognóstico depende, fundamentalmente, do tempo decorrido entre o diagnóstico e o tratamento, sendo que a mortalidade relatada na literatura pode ser de até $92 \%$ nos casos não tratados e de $60 \%$ nos tratados tardiamente. ${ }^{(4)}$

A ruptura pós-emética do esôfago representa de 10 a 15\% dos casos de perfuração do esôfago torácico, sendo a terceira causa mais freqüente desta ocorrência. ${ }^{(2)} 0$ seu diagnóstico é particularmente difícil e geralmente retardado pelo fato de a doença ser rara e ser confundida com outras entidades clínicas graves porém mais comuns nas emergências. ${ }^{(1)}$ A suspeita clínica com relação a esta entidade é fundamental para o diagnóstico diferencial. 0 caso do nosso primeiro paciente foi diagnosticado e tratado inicialmente como infarto agudo do miocárdio e, posteriormente, como embolia pulmonar com base no quadro clínico e radiológico apresentado. Somente após a realização de uma toracocentese com saída de líquido pleural suspeito e da informação de que o paciente havia vomitado excessivamente antes do início do desconforto torácico é que se considerou a possibilidade de ruptura esofágica, a qual foi confirmada pela toracotomia.

0 segundo paciente apresentava quadro clínico de enfisema mediastinal e subcutâneo de aparecimento espontâneo sem comprometimento hemodinâmico ou dor torácica como o primeiro paciente, o que nos fez pensar primeiramente em pneumomediastino espontâneo, que é uma condição benigna. ${ }^{(5,6)} A$ presença de derrame pleural 
à esquerda associada a uma toracocentese suspeita e à história pregressa de vômitos e alcoolismo nos levou à realização de um esofagograma e ao diagnóstico de ruptura esofágica.

0 terceiro paciente apresentava todo o quadro sugestivo de ruptura espontânea do esôfago, confirmado pela quantidade elevada de amilase no líquido pleural. A esofagoscopia não somente confirmou o diagnóstico como também visualizou o local da perfuração, ajudando inclusive na escolha do tipo e local da incisão.

Na quase totalidade dos casos de ruptura nãotraumática do esôfago, a começar pelo caso original de Boerhaave, observa-se freqüentemente a associação com vômitos, alcoolismo e excesso de ingestão alimentar. 0 ato de vomitar, particularmente quando repetido, poderia levar à incoordenação na abertura reflexa dos esfíncteres esofagianos. Esta disfunção resultaria em um aumento súbito e de grande magnitude na pressão intraluminal do esôfago, levando-o à ruptura na sua região mais frágil, que é a parede lateral esquerda. ${ }^{(7)}$

0 tratamento recomendado para esta condição é a toracotomia com sutura primária da lesão, quando factível, com ou sem reforço local; o debridamento mediastinal e a drenagem pleural; a gastrostomia para derivar a secreção gástrica e, ocasionalmente, a jejunostomia para suporte nutricional. ${ }^{(8)}$ Outras opções de tratamento recomendadas são a esofagectomia com ou sem reconstrução imediata do trânsito faringo-gástrico ${ }^{(9)}$; a exclusão funcional do esôfago pela esofagostomia cervical, a cerclagem da cárdia e a gastrostomia; ou a sutura da lesão associada ao uso de tubo em "T", à semelhança da drenagem das vias biliares. Estes últimos procedimentos são geralmente empregados em situações de maior gravidade, na presença de grande comprometimento dos tecidos locais, em casos de retardo no diagnóstico ou na impossibilidade do reparo primário..$^{(2,10,11)}$ 0 nosso primeiro paciente foi submetido à esofagectomia subtotal, que é um procedimento radical, devido à extensa necrose na parede do esôfago e no mediastino, o que talvez tenha contribuído para sua sobrevivência diante daquela situação. Nos outros dois pacientes, que apresentavam condições locais mais favoráveis, foi realizado o reparo primário. Porém, um deles evoluiu para óbito por choque séptico refratário no pós-operatório imediato.
Concluímos que a ruptura pós-emética do esôfago é um quadro grave cujo diagnóstico é comumente negligenciado na avaliação inicial. A presença de vômitos, derrame pleural esquerdo, toracocentese com aspiração de líquido suspeito de secreção digestiva e elevação da amilase foram elementos importantes para o diagnóstico e a indicação do tratamento cirúrgico. A evolução pós-operatória é quase sempre complicada por falências orgânicas e requer suporte intensivo. Como era de se esperar, a mortalidade e a morbidade são elevadas.

\section{Agradecimentos}

Agradecemos aos Drs. Sami Assi João, Kaliandre Medeiros e Andréa Fernandes Oliveira a participação no Caso 1.

\section{Referências}

1. Fell SC. Esophageal perforation. In: Pearson FG, Cooper JD, Deslauries J, Ginsberg RJ, Hiebert C, Patterson GA, et al., editors. Esophageal Surgery. New York: Churchill Livingstone, 2002. p. 615-36.

2. Jones WG, Ginsberg RJ. Esophageal perforation: a continuing challenge. Ann Thorac Surg. 1992;53(3):534-43.

3. Ximenes M, Silva RO, Vieira LF, Gregorcic A. The reversed gastric tube revisited: a useful esophageal replacement for benign disease. South Am J Thorac Surg. 1998;5(1):22-6.

4. Nemir P Jr, Wallace HW, Fallahnejad M. Diagnosis and surgical management of benign diseases of the esophagus. Curr Probl Surg. 1976;13(3):1-74.

5. Ralph-Edwards AC, Pearson FG. Atypical presentation of spontaneous pneumomediastinum. Ann Thorac Surg. 1994;58(6):1758-60.

6. Jougon JB, Ballester M, Delcambre F, Mac Bride T, Dromer CE, Velly JF. Assessment of spontaneous pneumomediastinum: experience with 12 patients. Ann Thorac Surg. 2003;75(6):1711-4.

7. Mackler SA. Spontaneous rupture of the esophagus; an experimental and clinical study. Surg Gynecol Obstet. 1952;95(3):345-56.

8. Whyte R 1, lannettoni MD, Orringer MB, Intrathoracic esophageal perforation. The merit of primary repair. J Thorac Cardiovasc Surg. 1995;109(1):140-4; discussion 144-6.

9. Orringer MB, Stirling MC. Esophagectomy for esophageal disruption. Ann Thorac Surg. 1990;49(1):35-42; discussion 42-3.

10. Abbott OA, Mansour KA, Logan WD Jr, Hatcher CR Jr, Symbas PN. Atraumatic so-called "spontaneous" rupture of the esophagus. A review of 47 personal cases with comments on a new method of surgical therapy. J Thorac Cardiovasc Surg. 1970;59(1):67-83.

11. Ximenes-Netto M, Silva R0, Fleury Jr 1. Perfuração do esôfago. Rev Bras Cir. 1982;72(6):351-8. 